\title{
A NOTE ON SEMIPRIME RINGS WITH DERIVATION
}

\author{
Dedicated to the memory of Professor H. Tominaga
}

\section{MOTOSHI HONGAN}

Tsuyama College of Technology

Numa, Tsuyama, Okayama 708

Japan

(Received September 26, 1995 and in revised form March 3, 1996)

\begin{abstract}
Let $R$ be a 2-torsion free semiprime ring, $I$ a nonzero ideal of $R, Z$ the center of $R$ and $d: R \rightarrow R$ a derivation. If $d[x, y]+[x, y] \in Z$ or $d[x, y]-[x, y] \in Z$ for all $x, y \in I$, then $R$ is commutative.
\end{abstract}

KEY WORDS AND PHRASES: Derivation, semiprime ring, 2-torsion free ring. 1991 AMS SUBJECT CLASSIFICATION CODES: $16 \mathrm{~W} 25,16 \mathrm{~N} 60$.

\section{INTRODUCTION.}

Throughout, $R$ will represent a ring, $Z$ the center of $R, I$ a nonzero ideal of $R$, and $d: R \rightarrow R$ a derivation. As usual, for $x, y \in R$, we write $[x, y]=x y-y x$ and $x \circ y=x y+y x$. Given a subset $S$ of $R$, we put $V_{R}(S)=\{x \in R \mid[x, s]=0$ for all $s \in S\}$. In [1], Daif and Bell showed that a semiprime ring $R$ must be commutative if it admits a derivation $d$ such that (i) $d[x, y]=[x, y]$ for all $x, y \in R$, or (ii) $d[x, y]+[x, y]=0$ for all $x, y \in R$. Our present objective is to generalize this result.

\section{THE RESULTS.}

As mentioned in $\S 1$, our present objective is to prove the following theorem which generalizes [1, Theorem 3$]$.

THEOREM 1. Let $R$ be a 2-torsion free semiprime ring, and let $I$ be a nonzero ideal of $R$. Then the following conditions are equivalent:

(1) $R$ admits a derivation $d$ such that $d[x, y]-[x, y] \in Z$ for all $x, y \in I$.

(2) $R$ admits a derivation $d$ such that $d[x, y]+[x, y] \in Z$ for all $x, y \in I$.

(3) $R$ admits a derivation $d$ such that $d[x, y]+[x, y] \in Z$ or $d[x, y]-[x, y] \in Z$ for all $x, y \in I$.

(4) $I \subseteq Z$.

In preparation for proving our theorem, we state the following two lemmas. 
LEMMA 1. Let $R$ be a semiprime ring, $I$ a nonzero ideal of $R$, and $a \in R$.

(1) Let $b \in I$. If $[b, x]=0$ for all $x \in I$, then $b \in Z$. Therefore, if $I$ is commutative, then $I \subseteq Z$.

(2) If $[a, x] \in Z$ for all $x \in I$, then $a \in V_{R}(I)$.

(3) Let $R$ be a 2-torsion free ring and $[a,[x, y]] \in Z$ for all $x, y \in I$, then $a \in V_{R}(I)$.

PROOF. (1) is well known.

(2) For any $x \in I$, we have $a[a, x]=[a, a x] \in Z$, and so we get $0=[a[a, x], x]=[a, x]^{2}$. Since $R$ is semiprime and $[a, x] \in Z$, we obtain that $[a, x]=0$ for all $x \in I$. Hence $a \in V_{R}(I)$.

(3) Since $Z \ni[a,[x, x y]]=[a, x[x, y]]=x[a,[x, y]]+[a, x][x, y]$ for all $x, y \in I$, we have $0=[a, x[a,[x, y]]+[a, x][x, y]]=2[a, x][a,[x, y]]+[a,[a, x]][x, y]$. Now, substituting $a x$ for $y$, we get $0=2[a, x][a,[x, a x]]+[a,[a, x]][x, a x]=2[a, x][a,[x, a] x]+[a,[a, x]][x, a] x=-2[a, x]^{3}-$ $2[a, x][a,[a, x]] x-[a,[a, x]][a, x] x$. Substituting $[x, y]$ for $x(y \in I)$, we have $2[a,[x, y]]^{3}=0$. Since $R$ is a 2-torsion free semiprime ring and $[a,[x, y]] \in Z$, we get $[a,[x, y]]=0$ for all $x, y \in I$. Hence we have $a \in V_{R}(I)$ by [1, Lemma 1$]$.

LEMMA 2. Let $R$ be a semiprime ring, $I$ a nonzero ideal of $R$, and $d: R \rightarrow R$ a nonzero derivation such that $d[x, y]+[x, y] \in Z$ or $d[x, y]-[x, y] \in Z$ for all $x, y \in I$. If $d(I) \subseteq V_{R}(I)$, then $I$ is commutative, and so $I \subseteq Z$.

PROOF. Let $a \in I$. For any $x, y \in I$, we have $0=[a, d[x, y] \pm[x, y]]= \pm[a,[x, y]]$, and so we get $a \in V_{R}(I)$ by [1, Lemma 1$]$. Therefore, $I$ is commutative, and so we obtain that $I \subseteq Z$ by Lemma 1 (1).

We are now ready to complete the proof of Theorem 1.

PROOF OF THEOREM 1. (1) $\Rightarrow(4)$. Let $d$ be a derivation such that $d[x, y]-[x, y] \in$ $Z$ for all $x, y \in I$. If $d=0$, then $I \subseteq Z$ by Lemma 1 (1) and (2). Now we suppose that $d \neq 0$. For any $x, y, z \in I$, we have $Z \ni d[x,[y, z]]-[x,[y, z]]=[d(x),[y, z]]+[x, d[y, z]]-$ $[x,[y, z]]=[d(x),[y, z]]+[x, d[y, z]-[y, z]]=[d(x),[y, z]]$, and so we have $d(x) \in V_{R}(I)$ by Lemma 1 (3), that is, $d(I) \subseteq V_{R}(I)$. Therefore we have $I \subseteq Z$ by Lemma 2 .

$(2) \Rightarrow(4)$. Let $d$ be a derivation such that $d[x, y]+[x, y] \in Z$ for all $x, y \in I$. Then the derivation $(-d)$ satisfies the condition $(-d)[x, y]-[x, y] \in Z$. And so we have $I \subseteq Z$ by (1).

$(3) \Rightarrow(4)$. For each $x \in I$, we put $I_{z}=\{y \in I \mid d[x, y]-[x, y] \in Z\}$ and $I_{x}^{*}=\{y \in I \mid$ $d[x, y]+[x, y] \in Z\}$. Then $I=I_{x} \cup I_{x}^{*}$. By Brauer's Trick, we have $I=I_{x}$ or $I=I_{x}^{*}$. By the same method, we can see that $I=\left\{x \in I \mid I=I_{x}\right\}$ or $I=\left\{x \in I \mid I=I_{x}^{*}\right\}$. Therefore, by (1) and (2) we have $I \subseteq Z$.

(4) $\Rightarrow(1),(4) \Rightarrow(2)$ and $(4) \Rightarrow(3)$ are clear.

The next is a generalization of $[1$, Theorem 2$]$.

COROLLARY 1. Let $R$ be a 2-torsion free semiprime ring, $Z$ the center of $R$ and $d: R \rightarrow R$ a derivation. If $d[x, y]+[x, y] \in Z$ or $d[x, y]-[x, y] \in Z$ for all $x, y \in R$, then $R$ is commutative.

PROPOSITION 1. Let $R$ be a 2-torsion free ring with identity 1. Then there is no derivation $d: R \rightarrow R$ such that $d(x \circ y)=x \circ y$ for all $x, y \in R$ or $d(x \circ y)+(x \circ y)=0$ for all $x, y \in R$.

PROOF. If there exists a nonzero derivation $d: R \rightarrow R$ such that $d(x \circ y)=x \circ y$ or $d(x \circ y)+(x \circ y)=0$ for $x, y \in R$, then we have $2 x=x \circ 1= \pm d(x \circ 1)= \pm 2 d(x)$ for all $x \in R$. Since $R$ is 2-torsion free, we get $d(x)= \pm x$ for all $x \in R$. For any $x, y \in R$, we have $x y+y x=x \circ y= \pm d(x \circ y)= \pm d(x y+y x)=2(x y+y x)$, and so we get $x \circ y=x y+y x=0$. Since $R$ is 2 -torsion free, we have $x^{2}=0$. Hence we have $0=x \circ(x+1)=2 x$, and so we 
get $x=0$ for all $x \in R$; a contradiction. If there exists a zero derivation $d: R \rightarrow R$ such that $d(x \circ y)=x \circ y$ or $d(x \circ y)+(x \circ y)=0$ for all $x, y \in R$, then we can easily see that $x=0$ for all $x \in R$; a contradiction.

REMARK. In Theorem 1 and Corollary 1, we can not exclude the condition "2-torsion free" as below.

EXAMPLE. We denote by $Z$ the integer system. Let $R=\left(\begin{array}{ll}Z / 2 Z & Z / 2 Z \\ Z / 2 Z & Z / 2 Z\end{array}\right), a=$ $\left(\begin{array}{ll}1 & 0 \\ 0 & 0\end{array}\right)$, and $d$ the inner derivation induced by $a$, that is, $d(x)=[a, x]$ for all $x \in R$. Then $R$ is a non-commutative prime ring with $\operatorname{char} R=2$, and $d[x, y] \pm[x, y] \in Z$ for all $x, y \in R$.

Finally, we state two questions.

Let $R$ be a 2-torsion free semiprime ring, $d: R \rightarrow R$ a nonzero derivation, and $I$ a nonzero ideal of $R$. And let $n$ be a fixed positive integer.

QUESTION 1. Does the condition that $d^{n}[x, y]+[x, y] \in Z$ or $d^{n}[x, y]-[x, y] \in Z$ for all $x, y \in I$ imply that $I \subseteq Z$ ?

QUESTION 2. Does the condition that $d^{m}[x, y]+d^{p}[x, y] \in Z$ or $d^{m}[x, y]-d^{p}[x, y] \in Z$ for some positive integers $m=m(x, y)$ and $p=p(x, y)$, and for all $x, y \in I$ imply that $I \subseteq Z$ ?

ACKNOWLEDGMENT. I wish to express my thanks to Professor H. Komatsu and the referee for helpfull sugestions.

\section{REFERENCE}

[1] DAIF, M.N. and BELL, H.E., "Remarks on derivations on semiprime rings," Internat. J. Math. \& Math. Sci. 15 (1992), 205-206. 


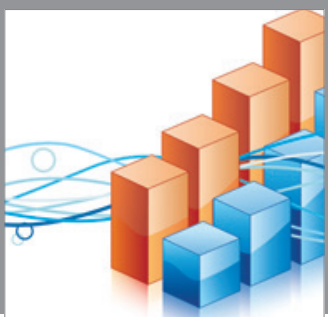

Advances in

Operations Research

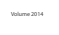

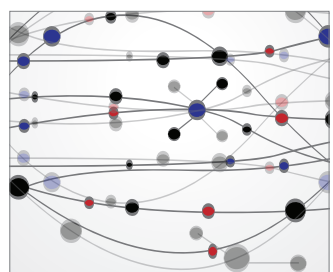

\section{The Scientific} World Journal
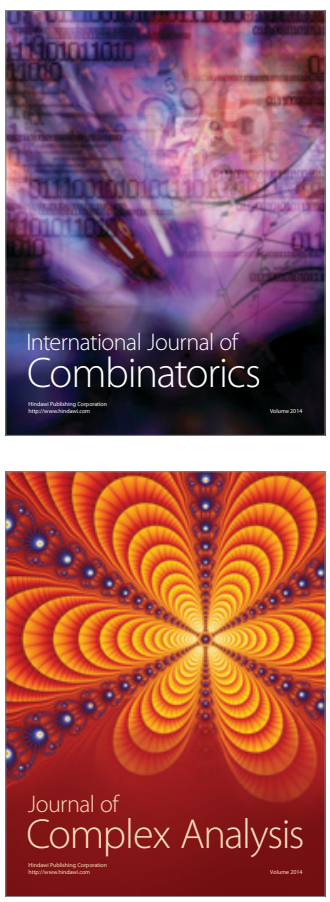

International Journal of

Mathematics and

Mathematical

Sciences
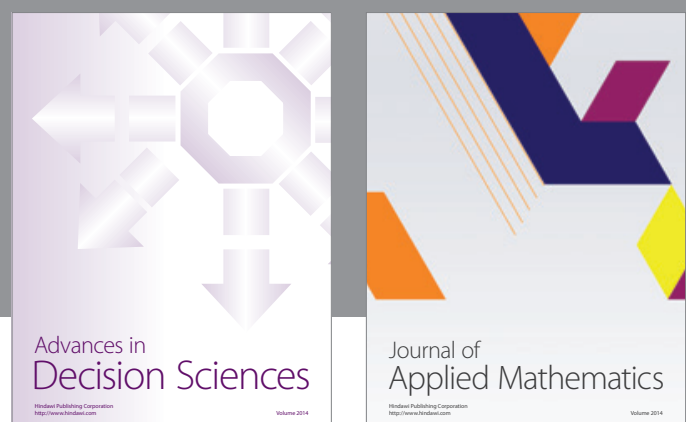

Journal of

Applied Mathematics
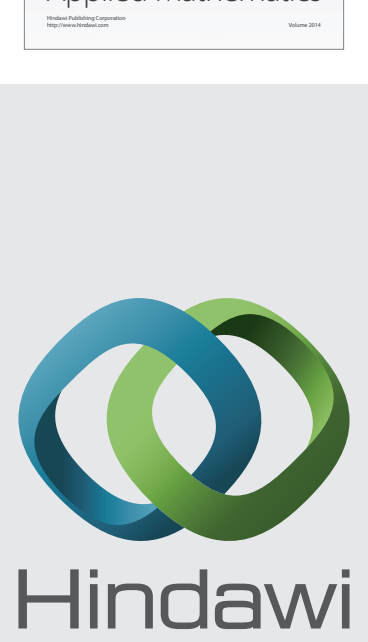

Submit your manuscripts at http://www.hindawi.com
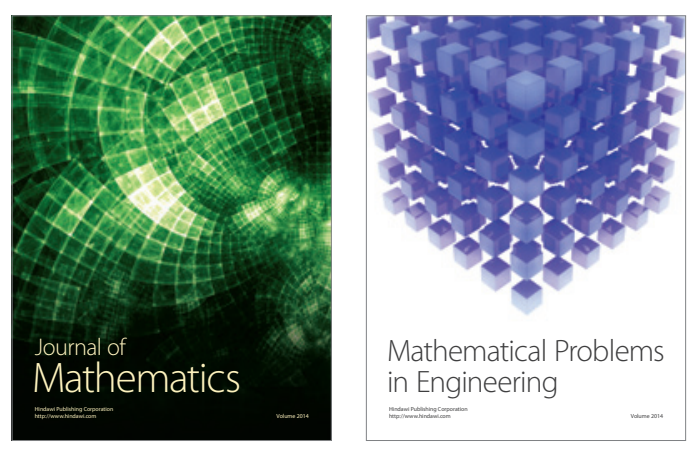

Mathematical Problems in Engineering
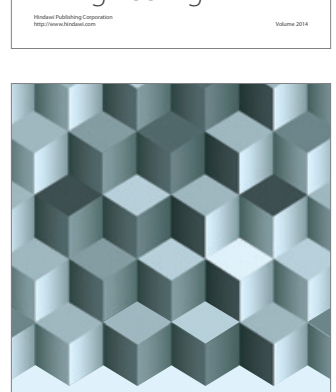

Journal of

Function Spaces
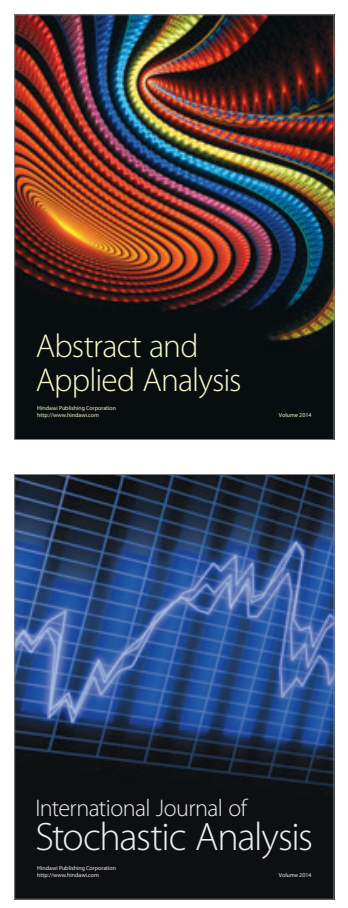

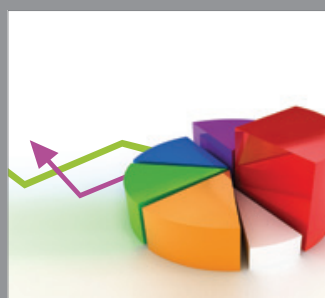

ournal of

Probability and Statistics

Promensencen
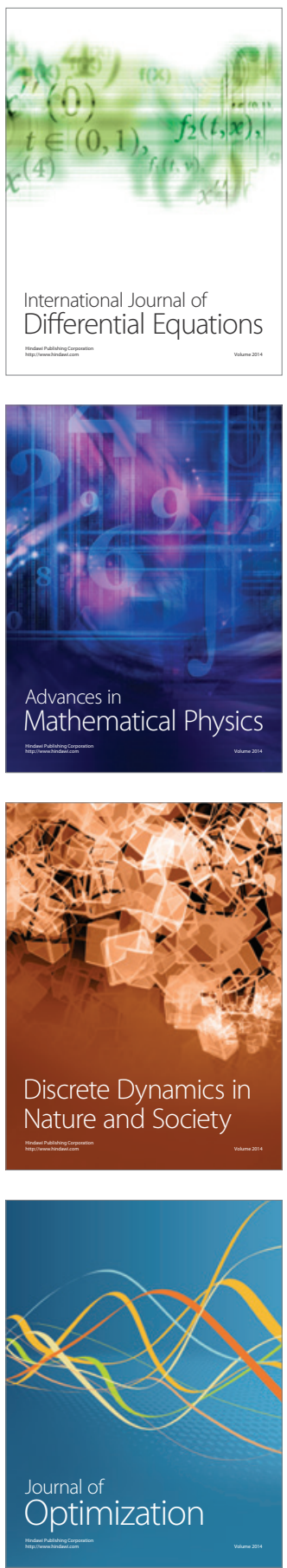